\title{
Lbl - Lstm : Log Bilinear And Long Short Term Memory Based Efficient Stock Forecasting Model Considering External Fluctuating Factor
}

\author{
Uma Gurav, S. Kotrappa
}

\begin{abstract}
Stock market prediction problem is considered to be NP-hard problem because of highly volatile nature of stock market. In this paper, effort has been made to design efficient stock forecasting model using log Bilinear and long short term memory (LBL-LSTM) considering external fluctuating factor such as varying Bank's lending rates. The external factor bank's lending rates affects stock market performance, as it plays vital role for the purchase of stocks in case of financial crisis faced by various business enterprises. Proposed LBL-LSTM based model shows performance improvement over existing machine learning algorithms used for stock market prediction.

Keywords : Data Mining, Artificial Intelligence, Stock Market Prediction, Long -Short Term Memory, Machine Learning Algorithms.
\end{abstract}

\section{INTRODUCTION}

Stock market forecasting is considered to be NP-hard problem as data generated is highly volatile and dynamic in nature. Hence there are no consistent patterns in data which effectively models the problem[1][14]. Moreover investments in stock markets is considered to be risky, but people expects better returns on investments(ROI). As global slowdown, unemployment is increasing, stock market investments is considered to be viable option for earning money for the people who are facing economic crisis.

Moreover, IPO is the capital investment of a commercial company or the investment of a single person in a company [2]. The stock market offers investment options for companies and brokers. The share price is mainly expected to determine future market trends for the company and from an investment point of view, profit sharing with investors. In the meantime, stock forecasting is a multivariate problem which is mainly characterized by various parameters, such as polynomial components [1], non-linearity and discontinuity, since variations occur due to external factors such as, political conditions, the country's economy conditions, variable bank interest rates and trader's expectation levels,

Revised Manuscript Received on April 25, 2020.

* Correspondence Author

Uma Gurav*, Assistant Professor, Department of CSE, K.I.T's College of Engineering, Kolhapur.

S. Kotrappa, Professor, Department of CSE, K L E's Dr MSS College of Engineering \& Technology, Belgaum.

(c) The Authors. Published by Blue Eyes Intelligence Engineering and Sciences Publication (BEIESP). This is an open access article under the CC BY-NC-ND license (http://creativecommons.org/licenses/by-nc-nd/4.0/) and current issues such as country suffering from COVID-19 disaster [2].Taking into consideration, the external fluctuating factor, investors who invest in stocks may need financial support from banks to purchase stocks. Although bank interest rates and the rise in the stock market is inversely proportional in relationship. A reduction in rates on bank loans for the purchase of shares by the Reserve Bank of India (RBI) leads to an increase in the stock market share rates and when RBI increases interest rates, the stock market goes down. RBI was shown to decrease the repurchase rate-REPO rate (key loan rate) by 35 basis points (bps) on August 7, 2019,this was the fourth time in 2019 when the repurchase rate was dropped by Reserve Bank of India (RBI) [5]. The Dow fell to 832 points on 10 October 2018, due to fears of higher interest rates [3]. Dow dropped significantly in March 2020 to near zero when the global corona virus pandemic crisis (covid-19) occurred [3].

As the economy slows down, the Federal Reserve lowers rates on federal funds to stimulate financial activity [3]. The bank's lower credit interest rates act as catalysts for financial economic growth, benefiting corporate loans, leading to higher returns, from investors and economists perspective [3]. The rise of the economy comes when consumers spend more, at lower interest rates, as they can afford to buy everything they need. Subsequently, companies can finance operations, expansions, acquisitions at a much cheaper rate and leading to multi fold profits., which leading to an increase in share prices [3]. As the study shows, a sharp rise in interest rates can slow down companies, leading to higher financing costs, but gradual increases may indicate positive trends in the overall economy [3] [5].

When investors faces financial shortfall ,banks are approached , private or commercial banks approach to the central Bank, RBI. Repurchase rate(Repo Rate) is the lending rate offered by $\mathrm{RBI}$ to various e-commerce banks and other financial institutions within the country[5]. Conversely, when the Fed raises interest rate in order to improve corporate earnings, some sectors often grows well, for example, entertainment, recreational, technology company stocks [3].

Hence in conclusion, to consider sudden decline in stock value due to external fluctuating factors such as Bank's lending rate is a crucial factor to be considered affecting stock market performance.

Time series data which is most important for financial research varies over time hence it needs to be analyzed and understand while considering external fluctuating factors such as bank's lending rates .

Published By:

Blue Eyes Intelligence Engineering DOI: 10.35940/ijeat.D8680.049420

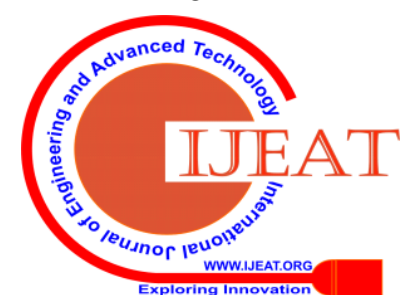


Similar to digital signal processing mathematical models, in the presence of noise, Particle filter and Kalman filters, kind of filtering approaches are applied to time series data [2][6][8].Volatility modeling is the Standard Deviation of asset returns and can be described as variations in Financial Time Series(FTS)[6].

\section{LOG BILINEAR- LONG SHORT TERM MEMORY MODEL (LBL-LSTM )MODEL}

Log Bilinear (LBL) model proposed by Mnih and Hinton, in 2009 is the very unique language model [9][24]. This feed-forward neural probabilistic model is simple with just one linear hidden layer and output layer[2][11][12]. LBL is used to model short term behavior of stock prices correctly[2], hence , stock buyers can determine when to buy and when to sell . Recurrent Neural Networks (RNN) can't learn short-term fluctuations in stock market [2]. Long Short-Term Memory (LSTM) networks are a type of RNN ,can model long term behavior pattern in time series based sequential prediction problems such as complex problem domains like machine translation, speech recognition etc.[17][20][22]. This work presents LBL based model with a hidden linear single layer .Hence it is a deterministic model[2].

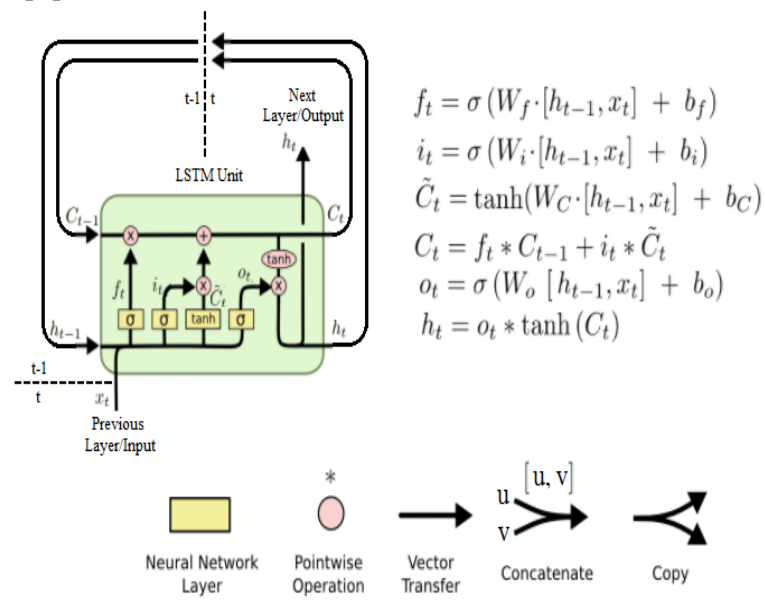

Fig. 1. Understanding LSTM Networks [13]

If a long and short term fluctuating FTS data is given for prediction LSTM suffer from carrying information from previous stage to the next stage. LSTM networks work better compared to traditional RNN since they overcome problem of 'Vanishing Gradient Problem'[10] . LSTM, in all cases , may not work well as they may leave some important part of information in short term dependencies ,hence LBL based model is the solution. LSTM fails to establish short term dependencies, although it works well in case of long term predictions, because as errors are back-propagated to multiple layers of LSTM, performance obtained is poor which can be improved by combining LBL. During training gradient becomes smaller and smaller. Here, gradients are calculated during back propagation. During Back propagation the derivatives of the network are calculated by moving outermost layer (close to output) back to initial layers. The chain rule is used during this calculation in which the derivatives from final layers are multiplied by the derivatives from early layers. The gradients keeps diminishing exponentially and therefore the weights and biases are no longer updated .

As discussed in our earlier research work [15] modified back propagation neural networks, in that case, gradients are the values used to update the weights of neural networks. The 'Vanishing gradient problem' is when the gradient slows down or decreases as it back propagates over time [20]. If a gradient value becomes extremely small, it doesn't contribute much to learning [20]. Hence learning rate considered here is considerably small 0.0001.It works on the formula given as :

new weight $=$ weight - learning rate*gradient

So in LSTM layers where it gets a small gradient update, it will stop learning[13][20]. Usually earlier layers don't learn[20], which leads to short-term memory loss problem. Hence proposed LBL-LSTM model can solve 'Vanishing Gradient ' problem which is the drawback of RNN while establishing long term relationships in time series forecasting models. Meanwhile mathematical model may get confused due to over fitting of parameters ,which may lead to reduced performance of the model .Hence, initially only one external fluctuating parameter is considered in this paper.

\section{The Research work contribution is as follows:}

- Efficient stock forecasting model LBL-LSTM is proposed ,by combining LBL with LSTM which models both short and long term behavior pattern of stock market respectively by feeding external fluctuating factors. External Fluctuating factor taken into account is, quarterly varying Bank's lending rate is one of the crucial parameter . (data obtained through official RBI website).

- This model's performance is evaluated on data obtained from yahoo.finanace.com. Results obtained proves, that performance of the proposed LBL-LSTM based stock forecasting model is better than existing machine learning based stock prediction models evaluated with respect to , Root mean square relative error (RMSRE) and training and testing accuracy.

Meanwhile , in our previous research work[2] RNN-LBL based model without considering external fluctuating factor for stock market predictions gives accuracy rate of $78 \%$. This research work is an extension to that work and crucial factors affecting stock performance are addressed.

\section{DESIGN OF PROPOSED LBL -LSTM MODEL}

\section{A. statement and System model}

In this research work, aim is to forecast stock price of a company, one step ahead, based on historical data.

Published By:

Blue Eyes Intelligence Engineering

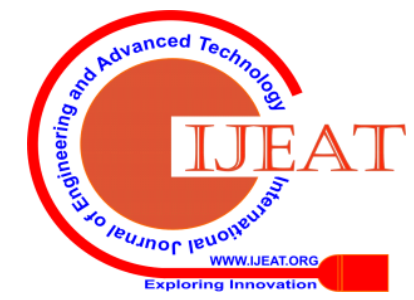


TABLE I: Bank's lending rate (Source : RBI) Lending rates of scheduled commercial banks[4]

\begin{tabular}{|l|c|c|c|c|}
\hline \multicolumn{1}{|c|}{ Month } & $\begin{array}{c}\text { Public Sector } \\
\text { Banks }\end{array}$ & $\begin{array}{c}\text { Private } \\
\text { Sector Banks }\end{array}$ & $\begin{array}{c}\text { Foreign } \\
\text { Banks }\end{array}$ & All SCBs \\
\hline Mar-2014 & 11.99 & 12.43 & 12.32 & 12.11 \\
\hline Jun-2014 & 11.95 & 12.55 & 12.32 & 12.10 \\
\hline Sep-2014 & 11.74 & 12.54 & 11.68 & 11.90 \\
\hline Dec-2014 & 11.68 & 12.34 & 12.01 & 11.84 \\
\hline Mar-2015 & 11.61 & 12.24 & 11.84 & 11.76 \\
\hline Jun-2015 & 11.46 & 12.07 & 11.69 & 11.61 \\
\hline Sep-2015 & 11.39 & 11.97 & 11.56 & 11.53 \\
\hline Dec-2015 & 11.14 & 11.85 & 11.33 & 11.31 \\
\hline Mar-2016 & 11.10 & 11.46 & 11.29 & 11.20 \\
\hline June-2016 & 11.13 & 11.47 & 11.11 & 11.23 \\
\hline Sept-2016 & 11.07 & 11.44 & 11.02 & 11.17 \\
\hline Dec-2016 & 11.04 & 11.23 & 11.01 & 11.09 \\
\hline Mar-2017 & 10.74 & 10.92 & 10.93 & 10.80 \\
\hline June-2017 & 10.59 & 10.82 & 10.87 & 10.67 \\
\hline Sep-2017 & 10.43 & 10.56 & 10.65 & 10.48 \\
\hline Dec-2017 & 10.29 & 10.47 & 10.62 & 10.36 \\
\hline Mar-2018 & 10.12 & 10.48 & 10.48 & 10.25 \\
\hline Jun-2018 & 10.13 & 10.53 & 10.36 & 10.26 \\
\hline Sep-2018 & 9.99 & 10.94 & 10.44 & 10.32 \\
\hline Dec-2018 & 9.94 & 11.11 & 10.66 & 10.35 \\
\hline Mar-2019 & 9.81 & 11.00 & 10.64 & 10.25 \\
\hline Jun-2019 & 9.82 & 11.11 & 10.59 & 10.31 \\
\hline Sep-2019 & 9.80 & 11.12 & 10.32 & 10.29 \\
\hline Dec-2019 & 9.60 & 11.06 & 9.94 & 10.14 \\
\hline Mathenatis & Fis & & 14 & \\
\hline
\end{tabular}

Mathematical Formulation of the given problem is :

Let , $\boldsymbol{X}_{\boldsymbol{t}}$ represents set of basic indicators. $Y$ denotes the stock's closing price value for short term interval at time $t$ described as follows:

$t=1,2, \ldots, T$

where $T$ is maximum lagging time. Given the historical parameters , $\boldsymbol{X}$ as described as in the equation

$X=\left\{X_{1}, X_{2}, \ldots, X_{T}\right\}$

previous close price value $\boldsymbol{Y}$ given as :

$\boldsymbol{Y}=\left\{\boldsymbol{Y}_{1}, \boldsymbol{Y}_{2}, \ldots, \boldsymbol{Y}_{T}\right\}$

Main objective is to predict $\boldsymbol{Y}_{T+1}$, the close price . Let us consider a set of stock market and

$u\left\{u_{1}, u_{2}, \ldots\right\}$,

set of stocks within stock market as follows:

$v=\left\{v_{1}, v_{2}, \ldots\right\}$

Research work considers stock market parameters, which composed of stock market basic indicators with $E x t_{l r}$ as Bank's lending rate.

Then, the output of the LBL-LSTM model is to predict future price of a particular stock within a stock market .
The LBL-LSTM model comprises of multiple input ,hidden and output layers, along with inner weight matrices. Hidden layer's activation parameters are:

$i_{l}^{v}=f\left(X i_{l}^{v}+D . s w_{i j}+E x t_{l r}\right)$

where, $\mathscr{t}_{\ell}^{V} \in \mathbb{S}^{e}$ represents the hidden layer illustration of stock $v$ at instance ( position) $\ell$ in time series, $s_{w} \in \mathbb{S}^{\theta}$ represents the hidden illustration of $\ell^{\text {th }}$ input stock of a particular stock market $v$. The activation function (AF) is represented by $f(t)$ and transition matrix (TM) of present stock is represented as follows[2]:

$\mathcal{D} \in \mathbb{S}^{e}$

and previous status is represented as follows

$W \in \mathbb{S}^{e}$

$\mathcal{D}$ stands for volatile stocks, short term behavior pattern of the stocks and $\mathcal{X}$ represents propagation of time series signals. The Eq. (6) is iteratively executed to calculate the updates in a time series sequence of each time instance. LSTM network architecture is shown in Fig. 1.

The next time instance, a predicted value can be derived using following equations, here $\mathrm{j}$ varies from 0 to $\sigma$

$i_{l}^{v}=\sum_{j}^{\sigma}\left(D_{j} \cdot s_{w_{l-j_{l}^{v}}} \bigoplus E x t_{l r}\right) \ldots(9)$

where $\mathcal{D}_{j} \in \mathbb{S}^{\oplus \times \in}$ illustrates the transition matrix for the particular time instance for short time behavior and $E x t_{l r}$, and $\sigma$ is the number of elements considered in a time series. The LBL model is shown in Fig. 2.
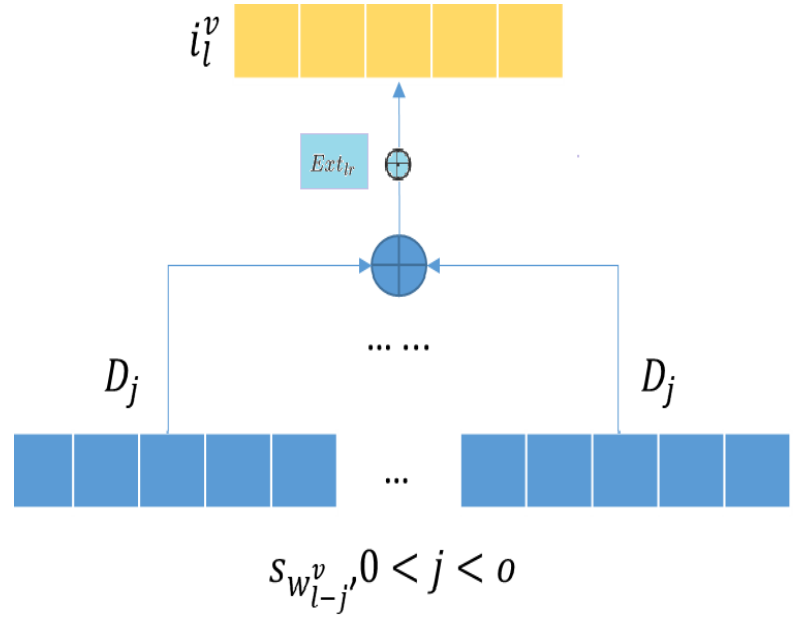

Fig. 2. LBL model Architecture considering external factor

In LBL, every position in the time sequence is modeled with a specific transition matrix[TM]. In general, LBL faces difficulty in learning the long-term behavior model efficiently [2] [19].

Published By:

Blue Eyes Intelligence Engineering

DOI: 10.35940/ijeat.D8680.049420

Journal Website: www.ijeat.org

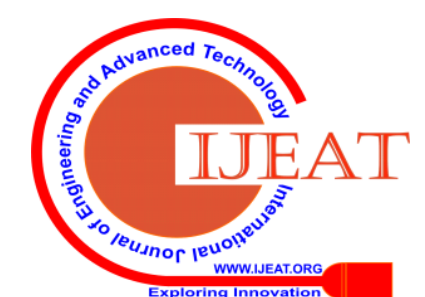


To address previous research issues on stock value prediction, this research presents a market behavior model that simultaneously captures the long and short term context and external parameters in historical data, rather than considering only one component in each hidden level ,unlike RNN. This working model considers multiple components in each hidden layer and adds position centered matrices in the LBL-LSTM structure, which is as described in Fig. 3. Consider a stock $v$, the hidden layered description of the stock at time instance $\ell$ in the sequence can be evaluated as:

$i_{l}^{v}=X i_{l-1}^{v}+\sum_{j}^{\sigma}\left(D_{j} \cdot s_{w_{l-j}^{v}} \bigoplus E x t_{l r}\right) \ldots$
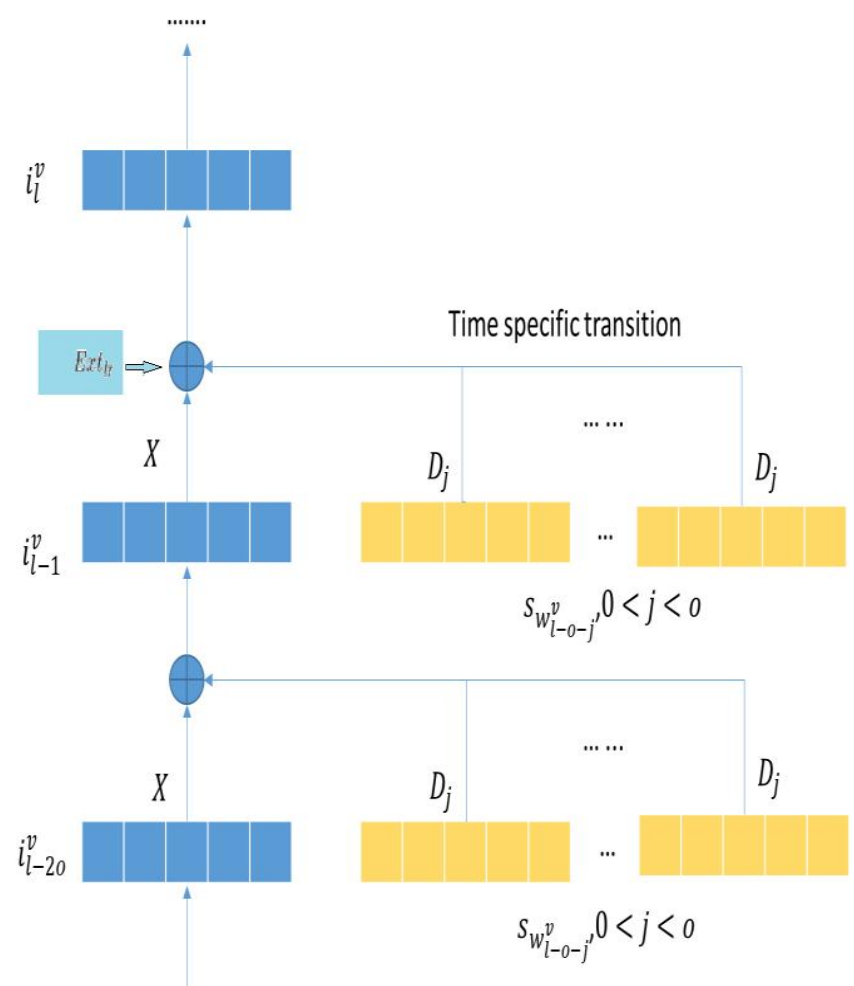

Fig. 3. Proposed LBL-LSTM based model architecture considering external factor

j varies from 0 to $\sigma$, where number of input stock values represented by $\sigma$ in each layer of proposed LBL-LSTM model, is called as adaptive dimension. The TM which is position-centric described as follows:

$\mathcal{D}_{j} \in \mathbb{S}^{\epsilon * \varepsilon}$

which is the factor for short term fluctuations, i.e. the $j^{\text {th }}$ score of the shares in each layer of the LBL-LSTM forecast model, the stock market behavior and the characteristics of the long-term history of the stock market. The values are learnt using LSTM . Furthermore, if we consider only one input for each layer and set the adaptive dimension $\sigma=1$, result of the LBL-LSTM model will be same like RNN neglecting the nonlinear activations. Important is that, for shorter patterns discoveries of adaptive dimension, the initial segment of a sequence, e.g. $\ell<0$. ,the equation. (12) can be reformulated as follows, $\mathrm{j}$ ranges from 0 to $\sigma$.

$$
i_{l}^{v}=X i_{0}^{v}+\sum_{j}^{\sigma}\left(D_{j} \cdot s_{w_{l-j}^{v}} \bigoplus E x t_{l r}\right) \ldots
$$

where $t_{0}^{v}=u_{0}$, showing the initial status of the stock market which will be the same, $v_{0}$ which addresses cold start problem (i.e., new companies venturing into market). Experiments are conducted to evaluate the outcome of LBL-LSTM model shows significant performance improvement in comparison to existing machine learning algorithms, which is experimentally proven in coming section

\section{COMPARATIVE ANALYSIS USING VARIOUS EXISTING MACHINE LEARNING (ML) ALGORITHMS AND MODELS :}

Input Parameters : This research work designed LBL-LSTM based model which considers stock market basic indicators such as open, volume, low, high, Adj. close parameter with $E x t_{l r}$ as Bank's lending rate. Here existing machine learning algorithms such as Recurrent Neural Networks based model, Logistic regression, Multilayer Perceptron model (MLP) for a time period from Dec .2013 to 2016 stock market prediction comparative analysis is done. Here $\mathrm{X}$-axis represents time duration, $\mathrm{Y}$ axis represents stock value of company.

I. Using Recurrent Neural Networks based model
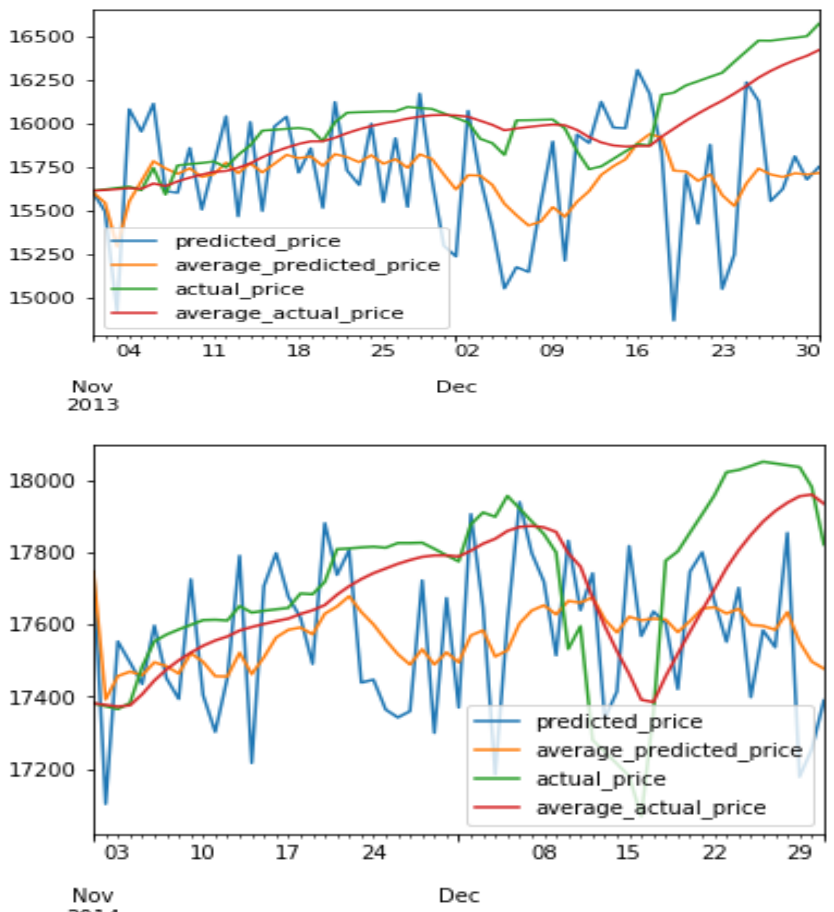

Nov
2014

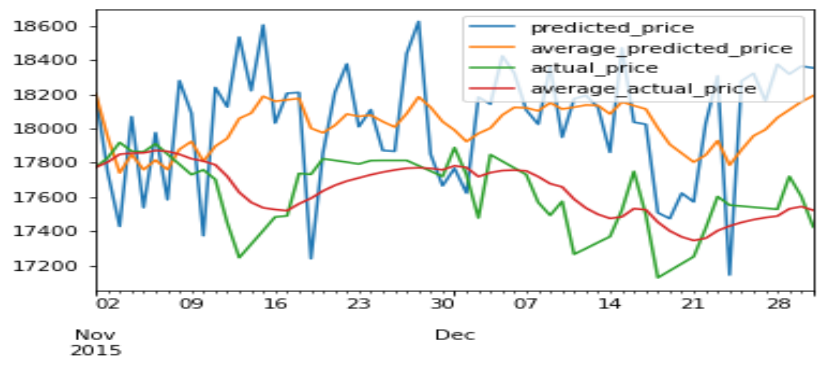

Published By:

Blue Eyes Intelligence Engineering

\& Sciences Publication

(C) Copyright: All rights reserved.

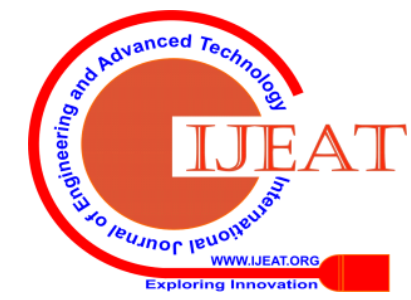



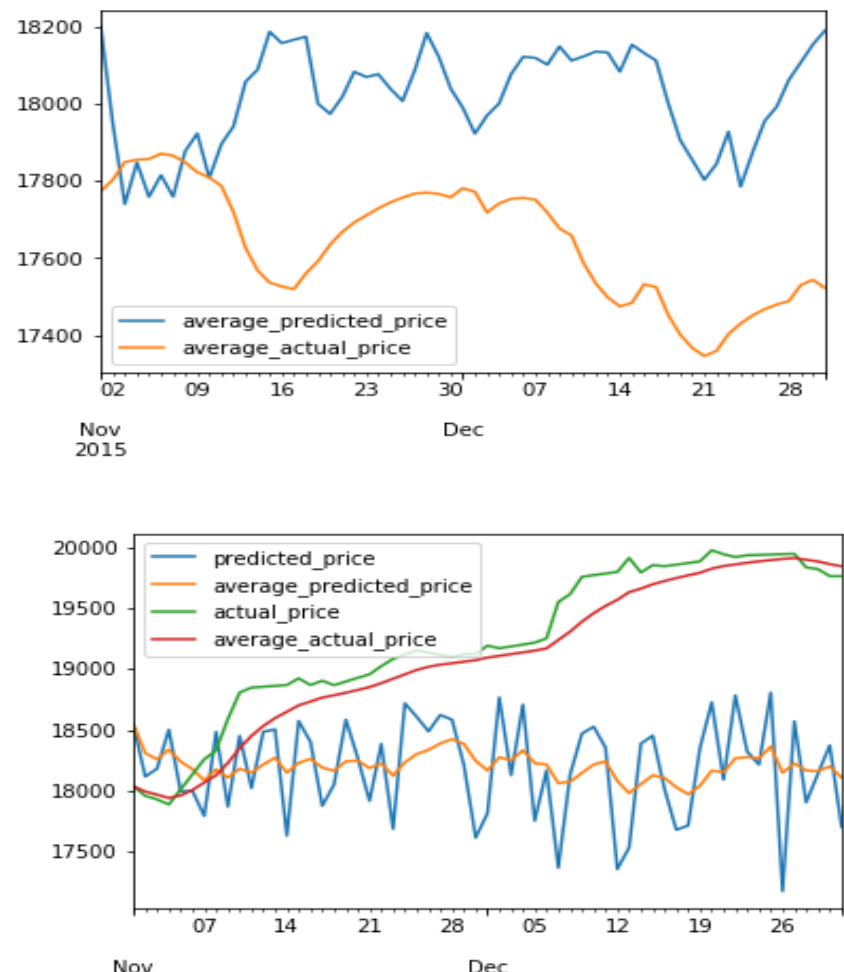

Nov
2016

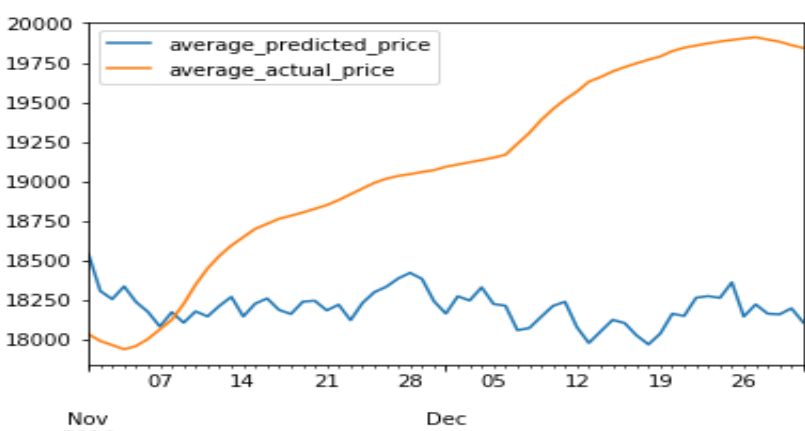

${ }_{2016}^{\text {Nov }}$

\section{Using Logistic regression}

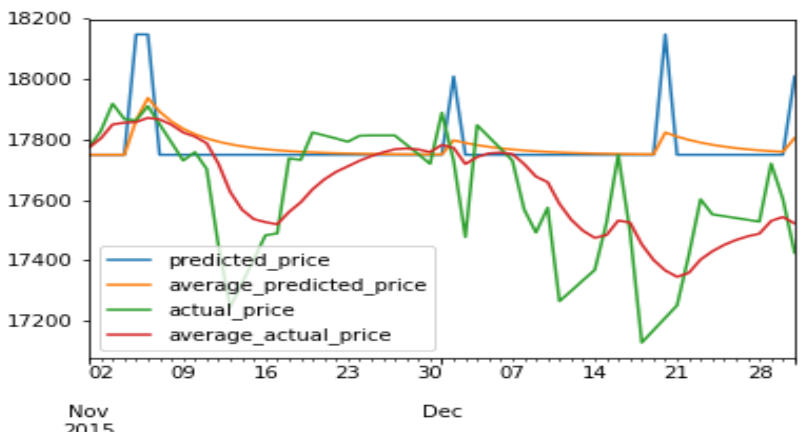

Nov
2015

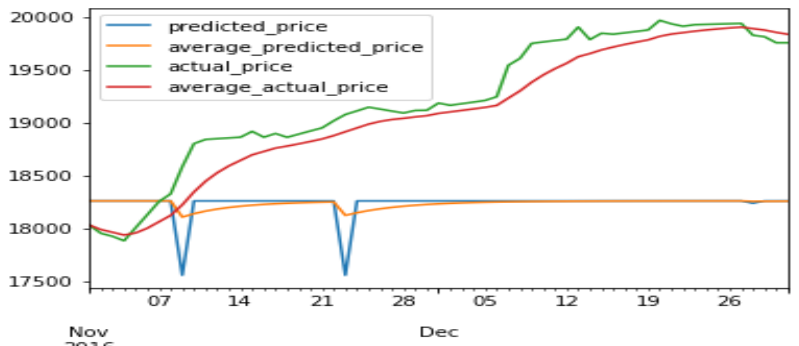

Nov
III. using MLP Classifier,

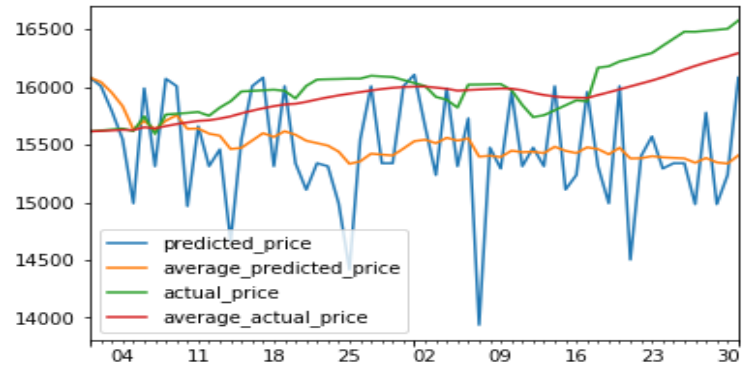

Nov

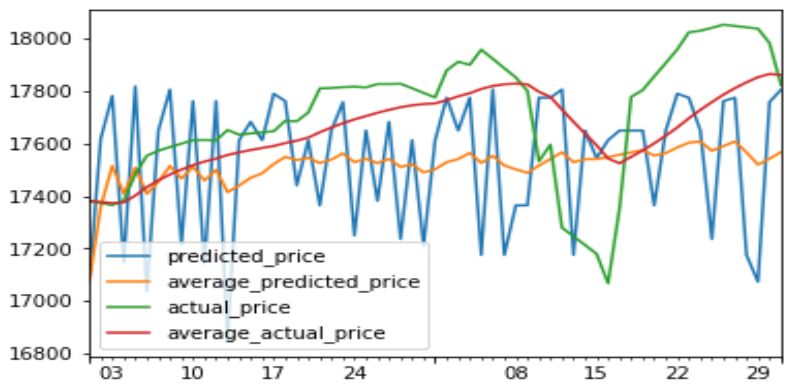

Nov

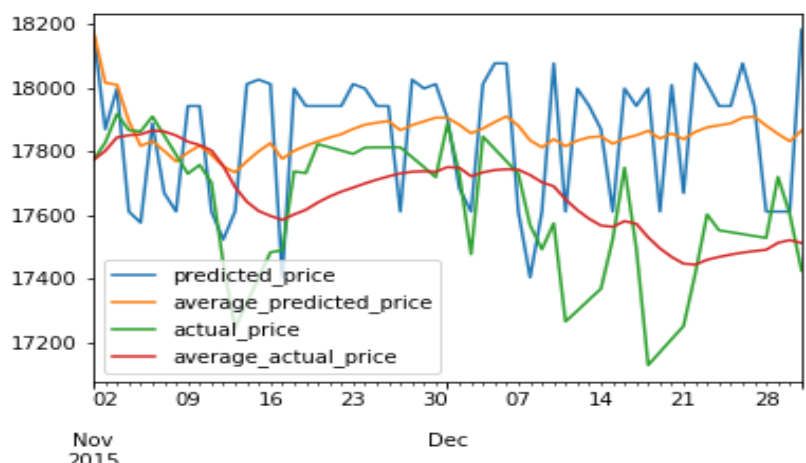

Nov

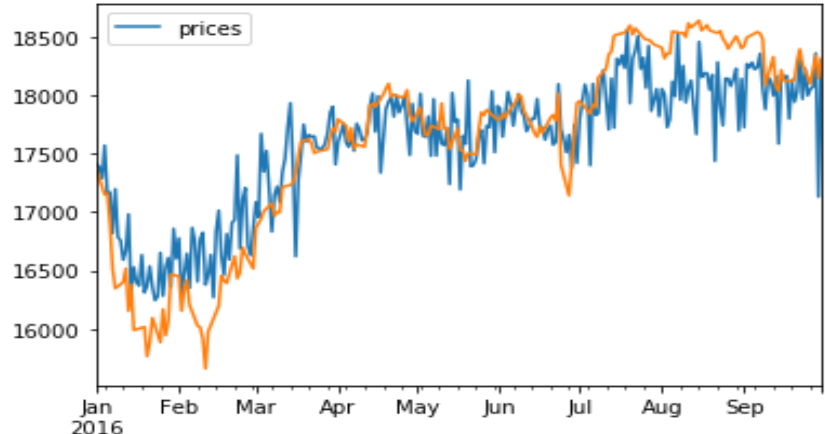

ublished By:

Blue Eyes Intelligence Engineering

\& Sciences Publication

(C) Copyright: All rights reserved.

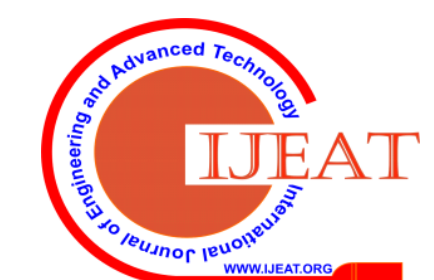


Lbl - Lstm : Log Bilinear And Long Short Term Memory Based Efficient Stock Forecasting Model Considering External Fluctuating Factor

\section{Implementation of LBL-LSTM model}

\begin{tabular}{|c|c|c|c|c|c|c|}
\hline \multirow{2}{*}{\multicolumn{7}{|c|}{ Volume Adj close }} \\
\hline & & & & & & \\
\hline 2012-01-03 & 58.928570 & 58.428570 & 58.485714 & 58.747143 & 75555200.0 & 50.994907 \\
\hline 2012-01-04 & 59.240002 & 58.468571 & 58.571430 & 59.062859 & 65005500.0 & 51.268970 \\
\hline $2012 \cdot 01 \cdot 05$ & 59.792858 & 58.952858 & 59.278572 & 59.718571 & 67817400.0 & 51.838169 \\
\hline 2012.01.06 & 60.392857 & 59.888573 & 59.967144 & 60.342857 & 79573200.0 & 52.380054 \\
\hline 2012.01.09 & 61.107143 & 60.192856 & 60.785713 & 60.247143 & 98506100.0 & 52.296970 \\
\hline
\end{tabular}

2019-12.24 284.890015 $282.920013 \quad 284.690002 \quad 284.269989 \quad 12119700.0 \quad 283.596924$ $2019 \cdot 12 \cdot 26 \quad 289.980011284 .700012 \quad 284.820007 \quad 289.910004 \quad 23280300.0289 .223602$ $\begin{array}{lllllll}2019-12.27 & 293.970001 & 288.119995 & 291.119995 & 289.799988 & 36566500.0 & 289.113831\end{array}$ $2019-12 \cdot 30 \quad 292.690002 \quad 285.220001289 .459991 \quad 291.519989 \quad 36028600.0290 .829773$ 2019.12.31 $293.679993 \quad 289.519989 \quad 289.929993 \quad 293.649994 \quad 25201400.0 \quad 292.954712$

\section{2 rows $\times 6$ columns}

Adam is adaptive learning rate algorithm used in training LBL-LSTM based optimization problems. Adam calculates individual learning rate for each parameter.

Considering Apple company's data from date 2012-2018 everyday's data used as training data set and 2019-2020 is used as Testing dataset. Here, Min-MaxScaler is used to normalize data in the range between $0 \& 1$. further ,reshaping of training and test data is done.

Results obtained in Python Jupyter editor Notebook are as follows:

$$
\text { close Predictions }
$$

\begin{tabular}{|c|c|c|}
\hline \multicolumn{3}{|l|}{ Date } \\
\hline 2018-05-29 & 187.899994 & 194.013824 \\
\hline 2018-05-30 & 187.500000 & 194.105957 \\
\hline 2018-05-31 & 186.869995 & 194.116745 \\
\hline 2018-06-01 & 190.240005 & 194.006668 \\
\hline 2018-06-04 & 191.830002 & 194.340866 \\
\hline$\cdots$ & $\cdots$ & $\cdots$ \\
\hline $2019-12-24$ & 284.269989 & 287.683411 \\
\hline $2019-12-26$ & 289.910004 & 288.832031 \\
\hline $2019-12-27$ & 289.799988 & 290.619995 \\
\hline $2019-12-30$ & 291.519989 & 292.389343 \\
\hline $2019-12-31$ & 293.649994 & 294.149414 \\
\hline
\end{tabular}

Published By:

Blue Eyes Intelligence Engineering

\& Sciences Publication

(C) Copyright: All rights reserved.

LBL- LSTM Model

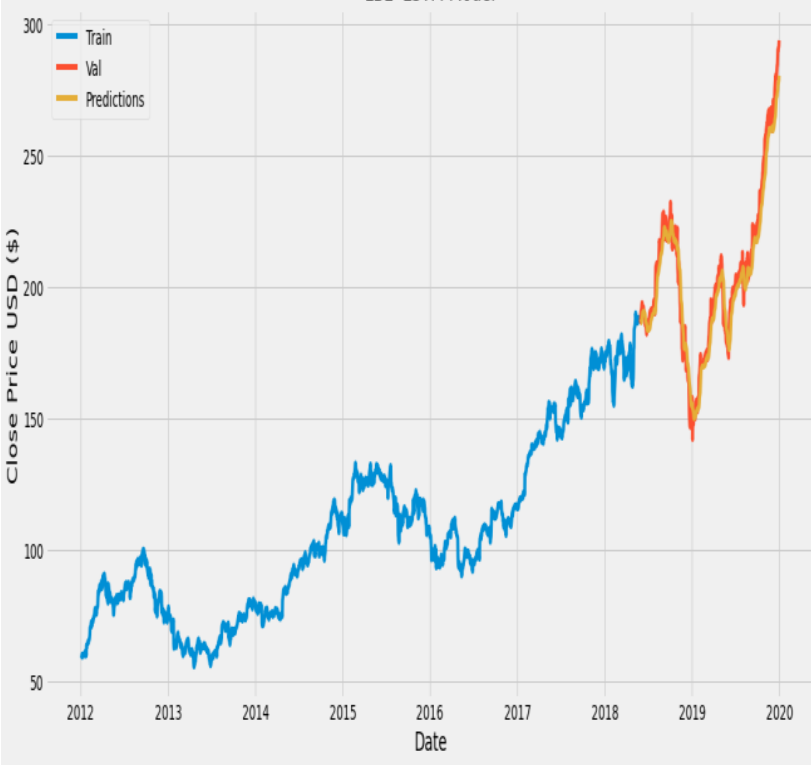

This section presents evaluation of LBL-LSTM based model over existing Machine learning algorithms used for stock market prediction . The proposed LBL- LSTM based stock forecasting model and existing algorithms are designed using python programming language.

Std . Avg. Method : This method allows to predict future stock prices as average of the previously observed values.

$$
x_{t+1}=1 / N \sum_{i=t}^{t} x_{i}
$$

The performance of proposed LBL -LSTM based model and existing machine learning based forecasting model is evaluated in terms of RMSRE and training and testing accuracy percentage .The experiments are conducted using yahoo.finance.com data sets and external bank's lending rate parameters. The Stock Exchange data ranges from January 1, 2012 to December 31 ${ }^{\text {st }}, 2019$.

Whereas, in this paper we considered RMSRE, which gives accurate performance evaluation. Let's consider that the total time interval the prediction is done as $T_{0}$. The actual stock price is described as $Y_{t}$ and forecasted value is described as $\hat{Y}_{t}$.

\section{ROOT MEAN SQUARED RELATIVE ERROR (RMSRE) METHOD}

The RMSRE is evaluated using following equation

RMSRE $=\sqrt{\frac{1}{T_{0}} \sum_{t=1}^{T_{0}}\left(\frac{\hat{Y}_{t+1}-Y_{t+1}}{Y_{t+1}}\right)^{2}}$.

This method takes squared errors between actual value and predicted value and taking average of it for all predictions. The lower RMSRE value, more accurate is the performance (i.e., it aggress with actual data). LBL-LSTM Model Score gives us $94 \%$ accuracy rate in terms of stock movement and price prediction. RMSRE error for LBL-LSTM standard averaging is 0.00418 .

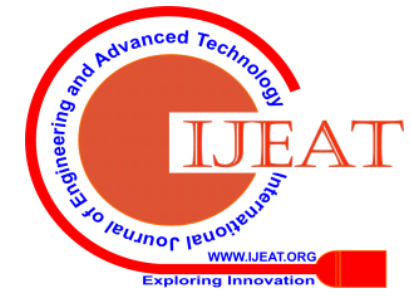


TABLE II: Comparative analysis of various machine learning algorithms (ML) with training and testing accuracy

\begin{tabular}{|l|l|l|}
\hline Method used & $\begin{array}{l}\text { Training } \\
\text { Accuracy } \\
\text { Percentage(\%) }\end{array}$ & $\begin{array}{l}\text { Testing } \\
\text { Accuracy } \\
\text { Percentage(\%) }\end{array}$ \\
\hline Regression & 45 & 44.33 \\
\hline MLP & 68.90 & 65.48 \\
\hline RNN & 71.79 & 71.77 \\
\hline LBL-LSTM & 94.51 & 94.33 \\
\hline
\end{tabular}

\section{CONCLUSION}

In this paper, we proposed LBL-LSTM based design of an efficient stock forecasting model which gives better performance than earlier research work .This work carried out extensive practical experimentation on various algorithmic methods such as Regression, Recurrent neural networks , Multilayer perceptron (MLP) classifier method and LBL-LSTM. Comparative analysis shows LBL-LSTM outperforms which gives $94 \%$ accuracy rate even considering fluctuating external market conditions such as quarterly varying bank's lending rates and reduced RMSRE is 0.00418 which is very less. In future ,more number of external fluctuating factors which affects short term and long tem behavior of stock forecasting will be considered thus trying not to over-fit and confuse the model with several parameters at a time.

\section{REFERENCES}

1. "A Novel Approach to Short-Term Stock Price Movement Prediction using Transfer Learning", Thi-Thu Nguyen, Seokhoon Yoon, Applied Sciences ,2019.

2. Uma Gurav, Dr.S.Kotrappa, "RNNLBL:A Recurrent Neural Network and Log Bilinear based Efficient Stock Forecasting Model ", IJITEE , Volume 9, Feb 2020

3. https://www.investopedia.com/investing/how-interest-rates-affect-sto ck-market/

4. Reserve bank of India ,lending rates of scheduled commercial banks (Excluding RRBS)

https://m.rbi.org.in/rbi-sourcefiles/lendingrate/LendingRates.aspx\#

5. https://www.bankbazaar.com/finance-tools/emi-calculator/current-rbi -bank-interest-rates.html

6. T. Rajbhandary, X.-P. Zhang, and F. Wang, "Piecewise constant modeling and kalman filter tracking of systematic market risk," Global Conference,(GlobalSIP), IEEE, Dec 2013.

7. G. H. Chen, K. Xiao, J. Sun, and S. Wu, "A double-layer neural network framework for high-frequency forecasting," ACM Transactions on Management Information Systems (TMIS,), vol.7 ,2018.

8. A. Wilson and Z. Ghahramani, "Copula processes," in Advances in Neural Information Processing Systems, pp. 2460-2468,2010.

9. Andriy Mnih, Geoffrey Hinton ,"A scalable hierarchical distributed language model",, NIPS'08: Proceedings of the 21st International Conference on Neural Information Processing Systems , ACM Digital Library, December 2008.

10. Indian Stock-Market Prediction using Stacked LSTM and Multi-Layered Perceptron, International Journal of Innovative Technology and Exploring Engineering (IJITEE), Volume-9 ,Issue-3, January 2020.

11. Andriy Mnih, Geoffrey Hinton, "Three New Graphical Models for Statistical Language Modeling", Machine learning, June 2007 ,Pages 641-648,https://doi.org/10.1145/1273496.1273577,ACM digital Library.

12. Frederic Morin , Yoshua Bengio",Hierarchical probabilistic neural network language model (2005)," AISTATS 2005

13. https://colah.github.io/posts/2015-08-Understanding-LSTMs/
14. Uma Gurav, Sidnal N." Predict Stock Market Behavior: Role of Machine Learning Algorithms", Intelligent Computing and Information and Communication. Advances in Intelligent Systems and Computing, vol 673. Springer, Singapore, 2018

15. Uma Gurav, Prof. Dr. Nandini Sidnal ," Adaptive Stock Forecasting Model using Modified BackPropagtion Neural Network (MBNN)",IEEE Xplore,,CTEMS' 2018

16. https://stackoverflow.com/understading-lstm

17. Uma Gurav, Dr. Nandini Sidnal ,"Opinion mining for reputation evaluation on unstructured Big Data". International Journal of Advanced Research in Computer Engineering ,2015

18. M. Göçken, M. Özçalıcı, A. Boru, and A. T. Dosdoğru, "Integrating metaheuristics and Artificial Neural Networks for improved stock price prediction," Expert Systems with Applications, vol. 44, pp. 320-331, 2016

19. Achyut Ghosh, Soumik Bose, Giridhar Maji , Narayan C. Debnath , Soumya Sen,"Stock Price Prediction Using LSTM on Indian Share Market," EPiC Series in Computing Volume 63, 2019, Pages 101-110 Proceedings of 32nd International Conference on Computer Applications in Industry and Engineering.

20. "Choosing the right Hyperparameters for a simple LSTM using Keras", https://towardsdatascience.com/choosing-the-right-hyperparameters-f or-a-simple-lstm-using-keras-f8e9ed76f046

21. Xiongwen Pang, Yanqiang Zhou , Pan Wang, Weiwei Lin and Victor Chang ,"Stock Market Prediction based on Deep Long Short Term Memory Neural Network", In Proceedings of the 3rd International Conference (COMPLEXIS 2018), 2019 SCITEPRESS - Science and Technology Publications

22. Raghav Nandakumar, Uttamraj K R, Vishal R, Y V Lokeswari, "Stock Price Prediction Using Long Short Term Memory", IRJET ,Volume: 05 ,Issue: 03 , Mar-2018

23. Harshal Patel , Shraddha Varman "Predicting Stock Prices Using LSTM", Murtaza Roondiwala1 ,volume 6 ,Issue 4, ,IJSR, April 2017

24. Mingoo Song, Chang D. Yoo. "Multimodal $27<10$ words representation: Kneser-ney smoothing/skipgram based neural language model" , 2016 IEEE International Conference on Image Processing (ICIP), 2016

\section{AUTHORS PROFILE}

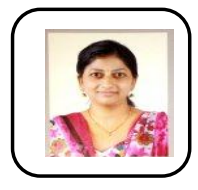

Mrs. Uma Gurav currently pursuing P.H.D in Computer Science and Engineering in V.T.U, Belgaum, INDIA. Presently working as a Assistant Professor in Department of CSE, K.I.T's College of Engineering, Kolhapur. Her Area of research includes Analysis of Algorithms, Data Sciences (AI/ML), Artificial Neural Networks, Deep Learning. She has Published several research papers in reputed International Journals .

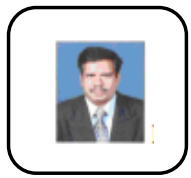

Dr. Kotrappa S. , Professor received Doctoral Degree (Ph.D) in Computer Science \& Engineering(CSE), from Walchand College of Engineering, Sangli, under Shivaji University Kolhapur, Maharashtra .He is presently working as a Professor, Department of CSE, K L E's Dr MSS College of Engineering \& Technology, Belgaum. His current research interests includes Big Data Analytics, Data Science (including AI/ML/DL), Block chain Technology and Software engineering (Agile, Design Patterns, OOMD, and AOSD/AOP).

Published By:

Blue Eyes Intelligence Engineering DOI: 10.35940/ijeat.D8680.049420

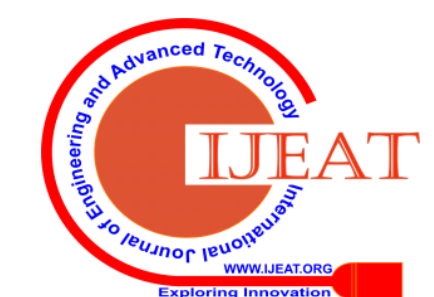

